\title{
HIGHLIGHTS
}

TARGETED THERAPIES

\section{Human CML stem cells insensitive to imatinib even when BCR-ABL is inhibited}

Treatment with the ABL kinase inhibitor imatinib is extremely effective and has induced complete responses in more than $80 \%$ of patients with chronic myeloid leukemia (CML). However, most patients experience disease recurrence after imatinib discontinuation, prompting the need for continued therapy that can result in high costs and the potential for long-term adverse effects. Recurrence occurs despite low to undetectable levels of BCR-ABL transcripts by reverse transcription PCR. The cause of disease persistence is unknown and it is unclear whether mechanisms central to leukemic stem-cell survival are dependent or independent of BCR-ABL activity. Understanding how CML stem cells evade the effects of ABL kinase inhibitors is crucial for disease eradication. Now, researchers have shown that primitive CML cells do not depend on BCR-ABL activity to survive, and therapies that target it, such as imatinib, may not eliminate CML stem cells.
To address the question of whether BCR-ABL is expressed and inhibited in cells that persist during treatment, "we took bone marrow samples from newly diagnosed patients with CML, isolated stem cells and cultured them with ABL kinase inhibitors. Cells that persisted in culture were analyzed for BCR-ABL expression and ABL kinase inhibition," explains Brian Druker, senior investigator of the study. The researchers demonstrated that innate resistance of the leukemic cells is likely to be BCR-ABL independent. "ABL kinase inhibitors were unable to kill all cells, consistent with what is seen in the clinic," Druker comments. They found that progenitor cells retained proliferative capacity despite continuous BCR-ABL inhibition.

The results of this study have farreaching implications for the use of agents that target $\mathrm{ABL}$ kinases, indicating that strategies that disrupt stem-cell renewal or the microenvironment are needed.

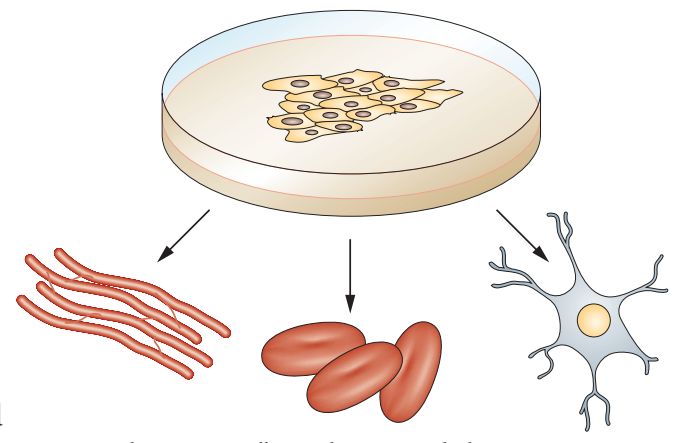

Druker notes: "ABL kinase inhibitors as single agents may not be capable of curing patients with CML." In the future, Druker's team plan to "identify agents that target stem cells that could be combined with ABL kinase inhibitors to eradicate disease."

\section{Lisa Hutchinson}

Original article Corbin, A. S. et al. Human chronic myeloid leukemia stem cells are insensitive to imatinib despite inhibition of BCR-ABL activity. J. Clin. Invest. 121, 396-409 (2011) 